\title{
Relationship between hip joint proximity area and sagittal balance parameters: an upright computed tomography study
}

\author{
Shunsuke Kikuchi ${ }^{1} \cdot$ Daisuke Nakashima $^{1} \cdot$ Yoshitake Yamada $^{2} \cdot$ Minoru Yamada $^{2} \cdot$ Yoichi Yokoyama $^{2} \cdot$ \\ Arihiko Kanaji ${ }^{1} \cdot$ Masaya Nakamura $^{1} \cdot$ Takeo Nagura $^{1} \cdot$ Masahiro Jinzaki $^{2}$
}

Received: 23 February 2020 / Revised: 9 October 2020 / Accepted: 10 November 2020 / Published online: 27 November 2020

(c) The Author(s) 2020

\begin{abstract}
Purpose A close relationship between sagittal spinal alignment and hip osteoarthritis (OA) has been documented. This study aimed to examine the relationship between hip joint proximity area and sagittal balance parameters in healthy subjects. Methods This prospective study enrolled 47 healthy volunteers who underwent 320-detector row upright computed tomography. Acquired data were reconstructed in a virtual three-dimensional space. The proximity area was determined by $<1 \mathrm{~mm}$ of the Hausdorff distance between the acetabulum and the femoral head. Volunteers were divided into the anterior and posterior proximity groups depending on the position of the closest area. Sagittal balance parameters [sagittal vertical axis (SVA), T1 spinopelvic inclination (T1-SPi), T1-pelvic angle, pelvic tilt, sacral slope, pelvic incidence, lumbar lordosis, thoracic kyphosis), offset distance between the centre of the acoustic meati (CAM) and C7 plumb line (CAM-C7-offset), and offset distance between the CAM and hip axis (HA) (CAM-HA-offset)] were compared between the two groups using independent sample $t$ test.

Results The anterior proximity group $(n=24)$ had higher SVA $(p=0.016)$ and T1-Spi $(p=0.015)$ than the posterior proximity group $(n=23)$. CAM-HA-offset was higher in the posterior than in the anterior proximity group $(p<0.000)$. There was no difference in other parameters $(p>0.05)$.

Conclusion The anterior proximity group had a positive anterior spinal balance; the posterior proximity group may have a more posterior gravity line than the hip joint centre. The anterior spinal balance may contribute to the anterior loading of the hip joint, with known relation with the initiation and onset of hip OA.
\end{abstract}

Keywords Hip osteoarthritis $\cdot$ Upright computed tomography $\cdot$ Sagittal balance parameters $\cdot$ Proximity area $\cdot 3 \mathrm{D}$ reconstruction

Electronic supplementary material The online version of this article (doi:https://doi.org/10.1007/s00586-020-06664-5) contains supplementary material, which is available to authorized users.

Yoshitake Yamada

yamada@rad.med.keio.ac.jp

$\triangle$ Takeo Nagura

nagura@z8.keio.jp

1 Department of Orthopedic Surgery, Keio University School of Medicine, Tokyo, Japan

2 Department of Diagnostic Radiology, Keio University School of Medicine, Tokyo, Japan

\section{Introduction}

Evaluation of the sagittal position of the spine and lower extremity during standing is an essential aspect when managing disorders of the spine and lower extremities. Analysis of sagittal balance while standing has been the clinical gold standard in the treatment of spinal deformity [1,2]. In addition, a close relationship between spinal deformity and hip osteoarthritis (OA), also known as 'hip-spine syndrome', can be an important factor in obtaining satisfactory clinical results of either spine or hip surgery [3, 4]. Pathology of the hip and its relation to the sagittal spinal and spinopelvic alignment has been widely analysed [5-9]. Recently, Day et al. examined the relationship between the severity of hip OA and sagittal spine deformity using standing full-body stereoradiography (EOS Imaging, Paris, France) [10]. This study showed that 
patients with severe OA had worse global sagittal alignment than those with limited OA. Thus, global sagittal alignment is crucial in surgical planning for hip surgery such as total hip arthroplasty [11]. Fukushima et al. examined the relationship between spinal sagittal alignment and acetabular coverage by evaluating centre edge angle only in 2D and demonstrated that the sacral slope and lumbar lordosis (LL) were greater in patients with developmental dysplasia of the hip (DDH) than in those with hip pain without DDH [12]. However, the relation between sagittal alignment and the distance of hip joint space in a healthy population is not well understood.

Femoroacetabular impingement (FAI) is considered a key pathological condition that causes hip OA and is usually caused by the abnormal contact between the femoral head-neck junction and the acetabulum $[13,14]$. As widely known, the severity of hip OA results in the superiorly and anteriorly migrated position of the femoral head in the acetabulum [15, 16]. Moreover, a previous study has shown the difference in spinopelvic alignment between FAI patients and asymptomatic subjects [17]. Thus, the sagittal alignment of the spine and pelvis can be another contributing factor to FAI. Grammatopoulos et al. conducted computed tomography (CT)-based quantitative assessments and revealed that symptomatic hips with FAI had a greater amount of supero-posterior coverage and individuals with symptomatic cam morphology had greater pelvic incidence (PI) [18]. This study suggested that the contact area of the hip and sagittal alignment may predispose patients to FAI and early hip OA; however, CT images were obtained in the supine position, which limited the analysis of the spinal and spinopelvic alignment. Moreover, previous studies have analysed the relationship between sagittal spinal alignment and hip pathologies in the standing position using two-dimensional (2D) radiography or full-body EOS [5, $6,8,10,19]$; however, the analysis of the three-dimensional (3D) distance of the hip joint space was not available, given the 2D analysis and the model were based on the skeletal morphology in the EOS system [20].

Therefore, this study aimed to examine the relationship between the proximity area of the hip joint and sagittal balance parameters in healthy subjects using upright $\mathrm{CT}$, which enabled us to obtain CT images of the whole body in the standing position using 320-detector row CT [21, 22]. Our study concentrated on the distribution of the proximity area of the acetabulum. We hypothesised that the position of the hip proximity area would correlate with the global sagittal malalignment in healthy subjects.

\section{Materials and methods}

\section{Study design and image acquisition}

This prospective study was approved by the institutional review board (\#20,160,384), and written consent was obtained from all participants. Altogether, 100 male or female volunteers aged 30-89 years who understood the purpose of the study were recruited from a volunteer recruitment company from June 2017 to March 2018. Individuals with a history of smoking, diabetes, hypertension, dyslipidaemia, awareness of dysuria, or those who had undergone surgery or were currently receiving treatment of the spine or lower extremity were excluded from the study.

All volunteers prospectively underwent upright CT (prototype TSX-401R, Canon Medical Systems Corporation, Otawara, Japan) (Fig. 1a). To avoid motion artefact and achieve a safe scanning condition, a single vertical pole was installed in the CT gantry (Fig. 2a). Volunteers were instructed to stand in a relaxed position, spread their legs according to their shoulder width, place their hands down, and touch their sacrum with the pole behind them, being careful not to swing the body during scanning. Scanning was performed at $100 \mathrm{kVp}$ and a gantry rotation speed of $0.5 \mathrm{~s}$ in the helical scan mode (80-detector row), with a noise index of 24 and a helical pitch of 0.8 for the body trunk. Image reconstruction was performed using adaptive iterative dose reduction 3D (Canon Medical Systems Corporation, Otawara, Japan), which could reduce the radiation [23].

\section{Radiographic analysis and intra- and interobserver reliability}

Sagittal balance parameters were measured using the RadiAnt ${ }^{\circledR}$ DICOM Viewer 4.6.9.18463 (Medixant, Poznan, Poland) (Fig. 2b). Global alignment parameters were sagittal vertical axis (SVA: horizontal distance from a C7 plumb line to the posterior edge of the sacral plate), T1 spinopelvic inclination (T1-SPi: the angle between the vertical plumb line and the line drawn from the vertebral body centroid of $\mathrm{T} 1$ to the centroid of the bicoxofemoral axis), and T1-pelvic angle (TPA: the angle between the line from the centroid of T1 to the femoral head axis and the line from the femoral head axis to the middle of the S1 endplate). Regional sagittal balance parameters included pelvic tilt (PT: the angle between the vertical plumb line and the line drawn from the midpoint of the sacral endplate to the centroid of the bicoxofemoral axis), sacral slope (SS), PI (the angle between a line from the bicoxofemoral axis to the midpoint of the sacral endplate and perpendicular to the sacral endplate), LL (the angle between the superior endplate of L1 and the superior sacral plate), thoracic kyphosis (TK: angle between the superior endplate of T4 and the inferior endplate of T12), offset distance between the centre of the acoustic meati (CAM) and C7 plumb line (CAM-C7-offset: the distance 
Fig. 1 Image acquisition and analysis. a Upright CT with 320 row-detector gantry. b Screenshot of AVIZO $®$ for reconstruction 3D model. c Analysing reconstructed 3D model using MeshLab®

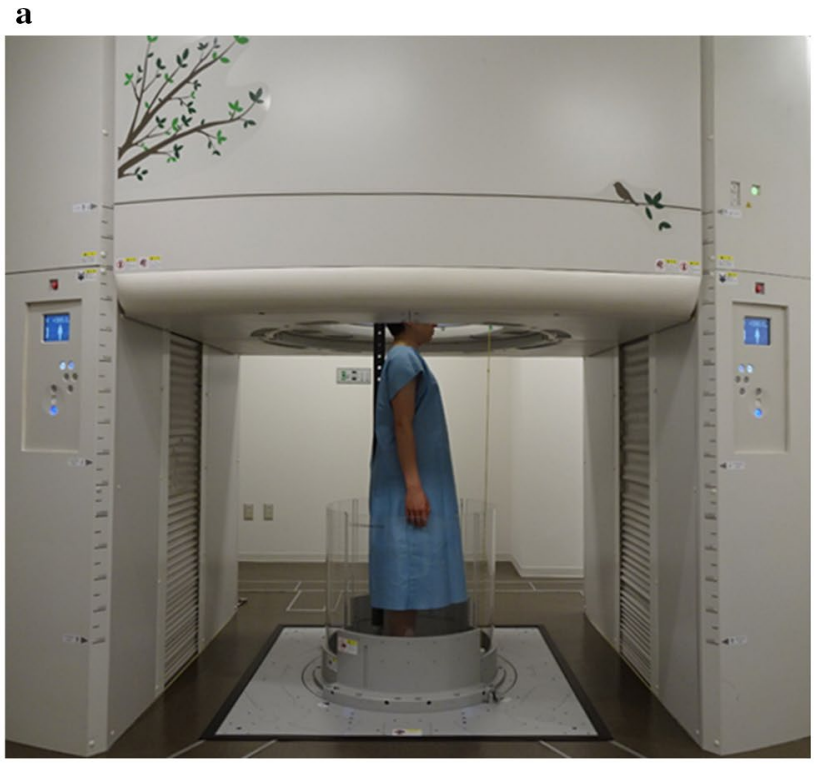

b

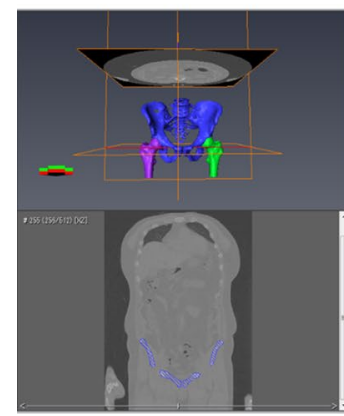

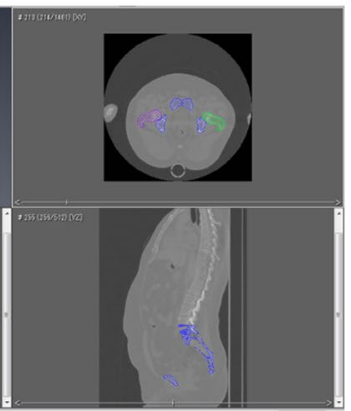

c

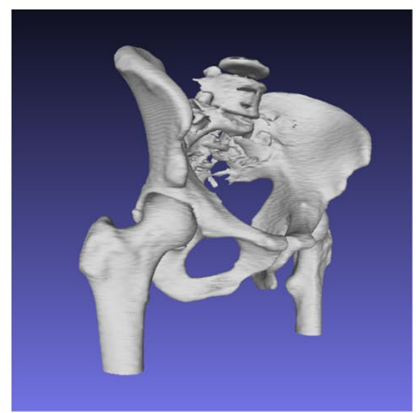

between a vertical line from CAM to $\mathrm{C} 7$ plumb line), and offset distance between CAM and hip axis (HA) (CAMHA-offset: distance between a vertical line from CAM to HA). A positive value of the sagittal balance parameters indicated 'anterior' balance, and a negative value indicated 'posterior' balance (Fig. 2a).

Two observers (hip joint surgeon with 10 years of experience and spine surgeon with 13 years of experience) measured the sagittal balance parameters, and the intra- and interobserver correlation coefficients were identified.

\section{D model evaluation of the proximity area of the hip}

The hip joint was reconstructed in the virtual 3D space using Avizo®9.0 (FEI Visualization Sciences Oregon, Hillsboro, OR, USA), and the proximity area was measured using
MeshLab_64 bit v1.3.3 (Visual Computing Lab of the ISTICNR, Pisa, Italy) (Fig. 1b,c). Hausdorff distances between the acetabulum and the femoral head were measured, and the area $1 \mathrm{~mm}$ below the Hausdorff distance was defined as the proximity area.

Hausdorff distance $(\mathrm{dH})$ is defined as the distance from any point in a given set $\mathrm{A}$ that can be reached at any point in another given set B by advancing at furthest. The 3D model in this study was constructed with polygons and, therefore, consisted of small triangles (Fig. 3a-c). Each vertex of triangle was defined as $\mathrm{A} 1, \mathrm{~A} 2$, and $\mathrm{A} 3$. The 3D model of the femoral head was defined as B (Fig. 3d). The shortest distance from A1 to set B was d1 (Fig. 3e), from A2 to set B was d2 (Fig. 3f), and A3 to set B was d3 (Fig. 3g). The maximum value of these $\mathrm{d} 1$, d2, and $\mathrm{d} 3$ was defined as $\mathrm{dH}$, so $\mathrm{d} 1$ was defined as $\mathrm{dH}$ (Fig. 3h). This $\mathrm{dH}$ meant the distance between the triangle and femoral head. 
a

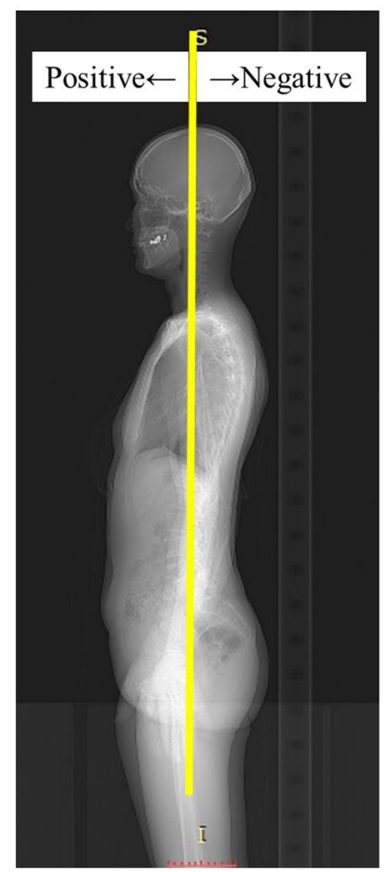

b

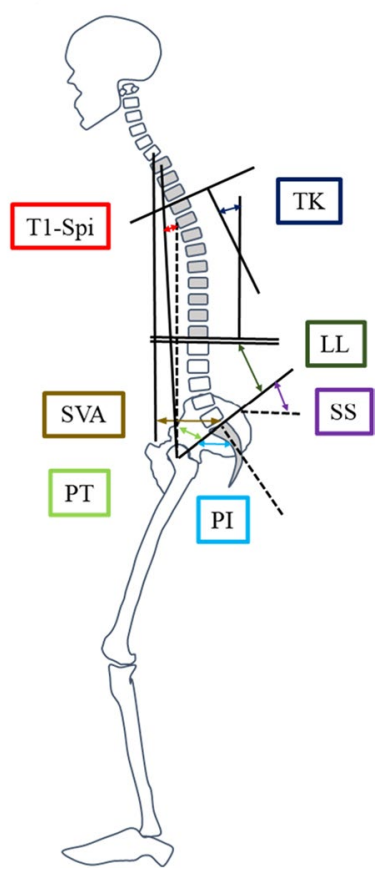

Fig. 2 Image of the lateral human body in the standing position. a Scout view of upright CT and a vertical line from CAM. b Illustration of the sagittal balance parameters $C T$ computed tomography, $C A M$ centre of the acoustic meati

For each triangle, the Hausdorff distance to the femoral head was measured and a threshold was set so that the area within the threshold distance could be detected, which we defined as the proximity area. There are no previous studies showing thresholds of the proximity area. In order to define the threshold, we examined various cut-off thresholds. Two representative cases are shown in Fig. 4. and Fig. 5. In case 1 , if the threshold was set at $1.5 \mathrm{~mm}$, almost the whole area of the acetabulum was included as the proximity area (Fig. 4). In case 2, if the threshold was set at $0.5 \mathrm{~mm}$, no area was included as the proximity area (Fig. 5). Thus, we decided on the threshold to be $1 \mathrm{~mm}$ to capture the differences depending on the cases.

We divided the volunteers into two groups: anterior proximity and posterior proximity. The acetabular location was defined by angles $\left(0^{\circ}\right.$ and $360^{\circ}$ : superiorly, $90^{\circ}$ : posteriorly, $180^{\circ}$ : inferiorly, $270^{\circ}$ : anteriorly). No previous studies have defined the anterior and posterior of the acetabulum. Some studies have expressed the acetabulum surface as a clock face, dividing it into $30^{\circ}$ [24]. From an anatomical perspective, the anterior edge of the posterior column is located around $60^{\circ}$. Thus, we defined the border between anterior and posterior as $60^{\circ}$. If the closest area of the acetabulum was located between $0^{\circ}$ and $60^{\circ}$ or between $180^{\circ}$ and $360^{\circ}$, the volunteer was assigned to the anterior proximity group. If the point was located between

$60^{\circ}$ and $180^{\circ}$, the volunteer was assigned to the posterior proximity group (Fig. 6).

\section{Statistical analysis}

All statistical analyses were performed using SPSS Statistics software version 25 (IBM Corp., Armonk, NY, USA). Sagittal balance parameters were compared between the anterior and posterior proximity groups using independent sample $t$ tests. Mean values are represented as mean \pm standard deviation. A $p$ value less than 0.05 was considered statistically significant.

\section{Results}

\section{Proximity area analysis}

Forty-five individuals who had significant contacts with the pole in standing and showed leaning posture on CT images and eight individuals who had obvious hip OA, scoliosis, or lumbosacral transitional vertebrae were excluded because such positions and conditions could affect the sagittal balance parameters. Finally, 47 healthy volunteers were analysed and divided into the anterior $(n=24)$ and posterior $(n=23)$ proximity groups as mentioned in the methods (Fig. 7). The characteristics of the two groups are shown in Table 1. The two groups did not differ in age, height, body weight, and body mass index (BMI) $(p>0.05)$.

\section{Radiographic analysis}

The intra- and interobserver reliabilities of the spinal and spinopelvic parameters were 0.915 (95\% confidence interval, 0.863-0.986) and 0.897 (95\% confidence interval, 0.847-0.993), respectively, so these data were highly reliable.

The sagittal balance parameters of the anterior and posterior proximity groups were compared, and the results are shown in Table 2. The two groups had similar morphology, as proven by their PI $\left(48.1^{\circ} \pm 7.2^{\circ}\right.$ vs. $50.1^{\circ} \pm 10.0^{\circ}$, $p=0.433$ ), and similar severity of thoracolumbar deformity, as proven by the TPA $\left(12.1^{\circ} \pm 5.9^{\circ}\right.$ vs. $12.0^{\circ} \pm 5.1^{\circ}$, $p=0.974)$.

Despite these similarities, the SVA $(23.5 \pm 18.9 \mathrm{~mm}$ vs. $11.6 \pm 13.5 \mathrm{~mm}, p=0.016)$ and T1-Spi $(-2.6 \pm 1.7 \mathrm{~mm}$ vs. $-3.9 \pm 1.8, p=0.015)$ were significantly higher in the anterior than in the posterior proximity group. In addition, CAMHA-offset $(5.8 \pm 16.8 \mathrm{~mm}$ vs. $24.7 \pm 15.6 \mathrm{~mm}, p<0.000)$ was significantly higher in the posterior proximity group than in the anterior proximity group. No difference was found in TK, LL, SS, PT, and CAM-C7-offset between the two groups $(p>0.05)$. 
Fig. 3 Hausdorff distance. a 3D model of the acetabulum. b Enlarged view of the 3D model. The 3D model consisted of small triangles. c Enlarged view of small triangles. d One triangle of the acetabulum was defined as set $\mathrm{A}$ (each vetex $\mathrm{A} 1, \mathrm{~A} 2$, and $\mathrm{A} 3$ ), and the $3 \mathrm{D}$ model of the femoral head was defined as set B (large blue circle). e The shortest distance between $\mathrm{A} 1$ and set $\mathrm{B}$ was $\mathrm{d} 1$. f The shortest distance between $\mathrm{A} 2$ and set $\mathrm{B}$ was $\mathrm{d} 2$. $g$ The shortest distance between A3 and set $\mathrm{B}$ was $\mathrm{d} 3$. h Comparing $\mathrm{d} 1, \mathrm{~d} 2$, and $\mathrm{d} 3$, the maximum shortest distance was A1. A1 was defined as $\mathrm{dH}$ (Hausdorff distance) a

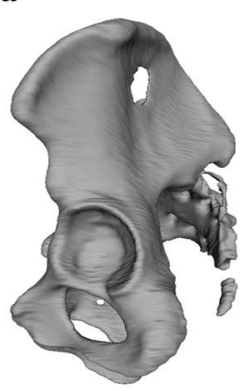

b

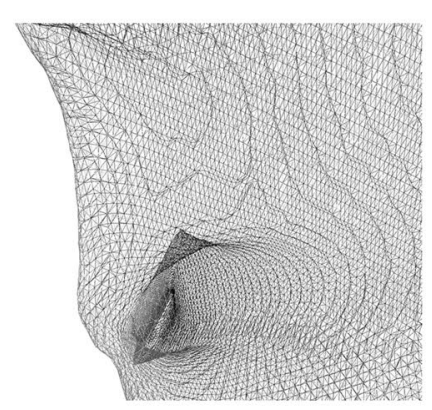

c

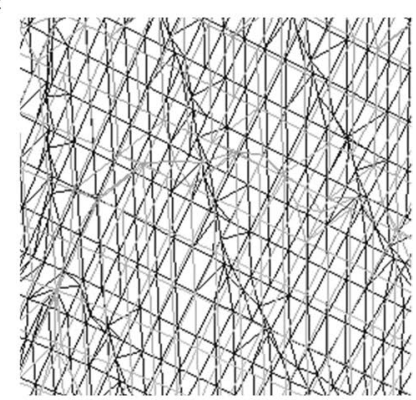

d

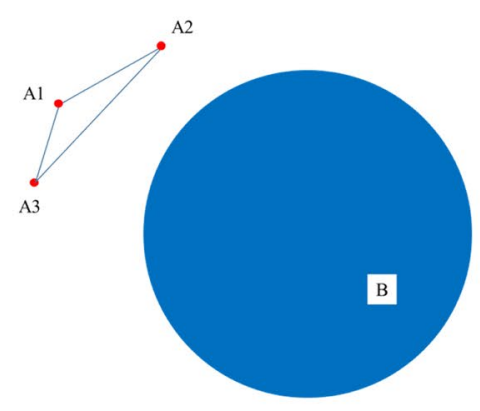

f

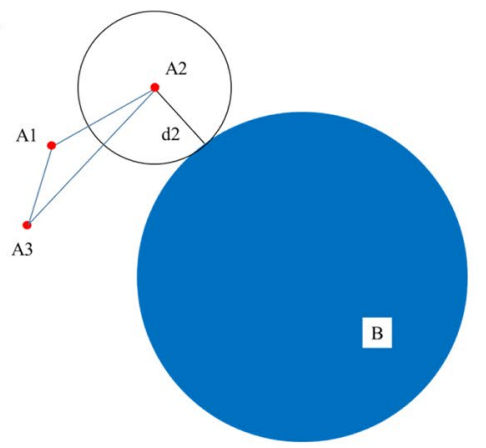

h

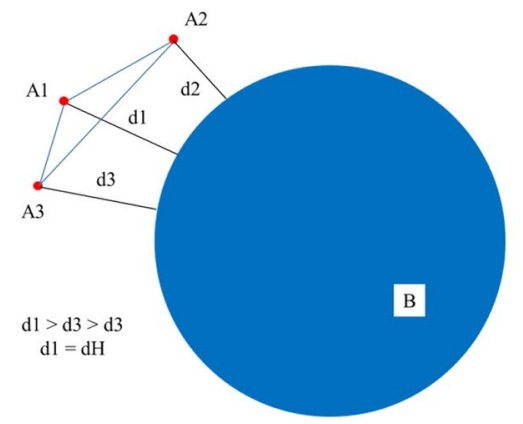

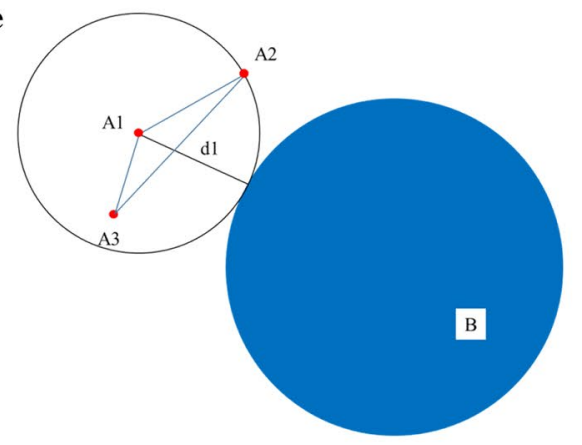

g

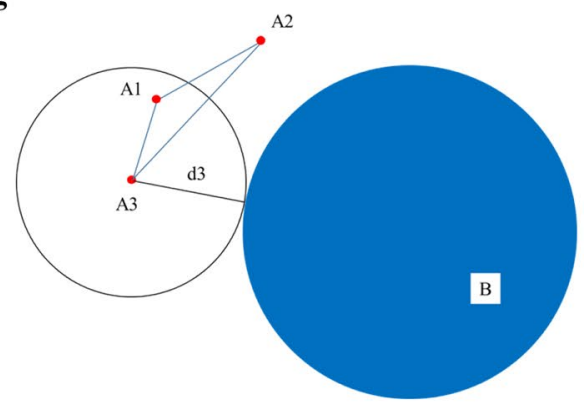

\section{Discussion}

To the best of our knowledge, this is the first study to evaluate the relationship between the 3D distance of the hip joint space and the sagittal alignment of the whole body in healthy subjects. Upright CT enabled us to evaluate both of these parameters simultaneously in a natural standing position. Our data showed that SVA and T1-Spi were significantly 
Fig. 4 Case 1. Threshold was set a $1.5 \mathrm{~mm}$. b $1 \mathrm{~mm}$. c $0.5 \mathrm{~mm}$. Proximity area is expressed as red area

Fig. 5 Case 2. Threshold was set a $1.5 \mathrm{~mm}$. b $1 \mathrm{~mm}$. c $0.5 \mathrm{~mm}$. Proximity area is expressed as red area

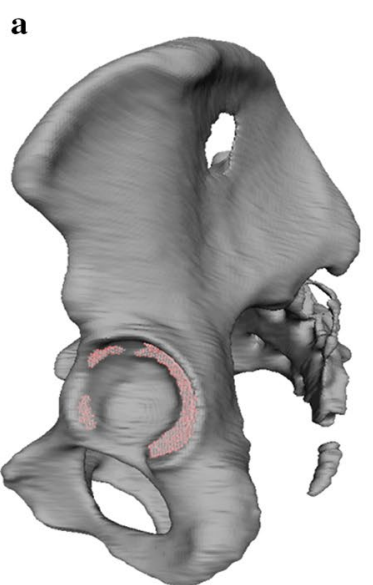

b

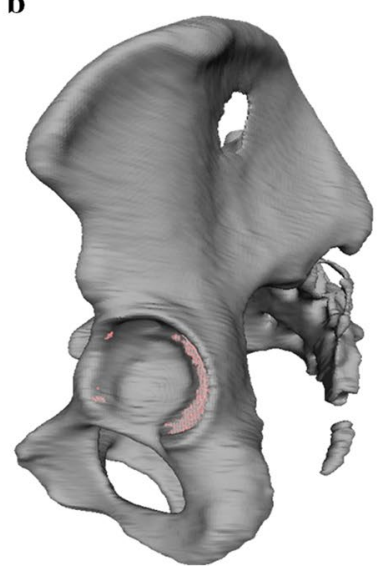

b

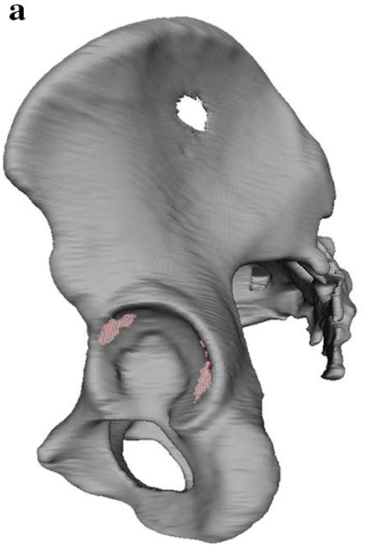

a

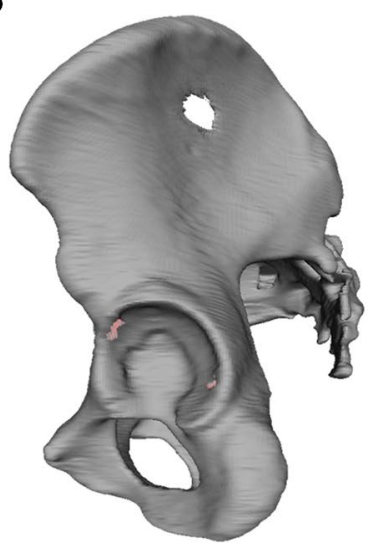

c
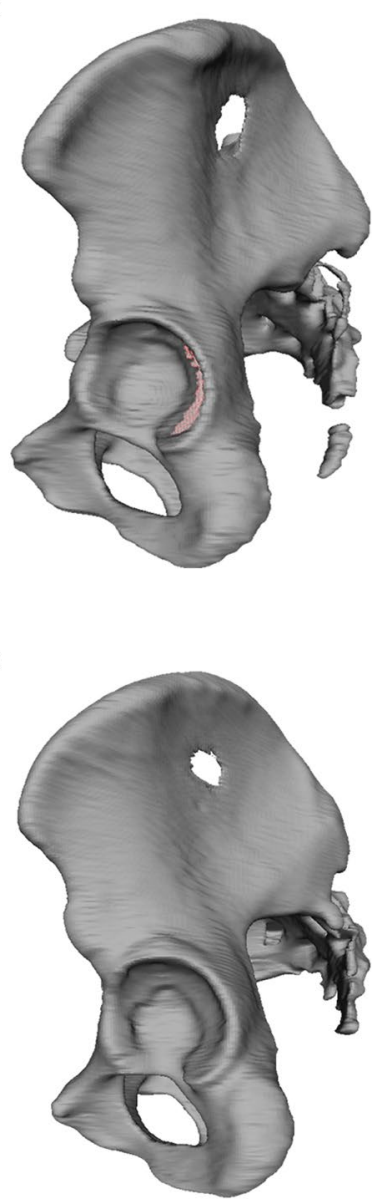

Fig. 6 Definition of the two groups. a Defined angles of the anterior and posterior proximity groups. b Definition image with the 3D model b

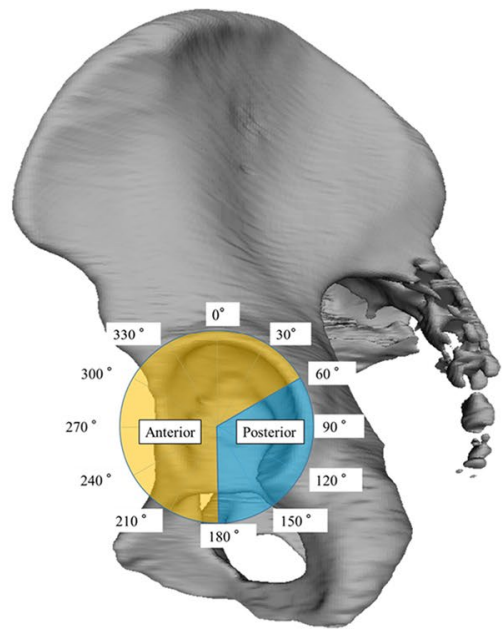

higher in the anterior proximity group than in the posterior proximity group. In addition, CAM-HA-offset was significantly higher in the posterior proximity group than in the anterior proximity group. SVA and T1-Spi are considered as key sagittal parameters in postural control in standing $[1,2]$. According to a previous study by Hasegawa et al. [25] who evaluated standing sagittal alignment in 136 healthy subjects, a vertical line from CAM was equivalent to the gravity line. Thus, our results indicated that the anterior proximity group had a positive anterior spinal balance and the posterior proximity group had a less anterior spinal balance with a more posterior gravity line with regard to the hip joint 
a

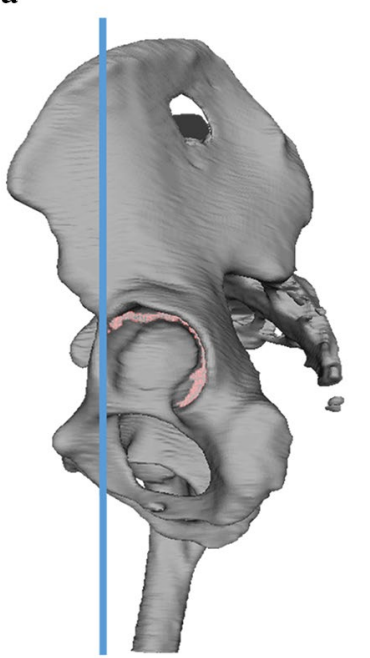

b

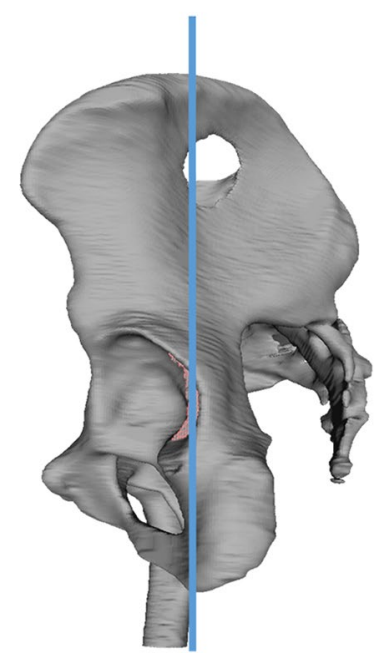

Fig. 7 Typical cases with anterior $\mathbf{a}$ and posterior $\mathbf{b}$ proximity area of the hip in standing. Proximity area is expressed as red area, and CAM is drawn as blue line

centre. The results revealed a close relationship between the sagittal spinal balance and the joint space distance of the hip and indicated that the position of the gravity line may affect the joint space distance. In contrast, no difference was found

in the thoracolumbar or spinopelvic parameters between the anterior and posterior proximity groups. These results suggested that the lumbosacral and pelvic alignment are nondominant factors when determining the joint space distance.

A clinical study by Day et al. showed a positive relationship between the sagittal spinal balance and severity of hip OA [10]. In particular, SVA and T1-Spi were higher in the severe OA group than in the limited OA group. Our results implied that the positive anterior sagittal balance and anterior position of the gravity line with respect to the hip joint centre induced the anterior loading of the hip joint. Although contact pressure is important in loading the acetabular surface, measuring pressure on the acetabular cartilage is difficult because of its invasiveness. Leunig et al. showed that the most frequent location for FAI was the anterosuperior rim area [14]. Considering this insight, we decided to measure Hausdorff distance to evaluate the location of the femoral head in the acetabulum in 3D. However, to our knowledge, no study has evaluated the relationship between global alignment and hip joint space distance in patients with hip OA. Although the anterior force of the hip is considered as an important factor for the onset and initiation of the hip joint pain [26], future clinical studies are required to reveal whether the anterior loading has any influence on hip pathologies such as FAI and early OA.
Table 1 Characteristic of the anterior proximity group and posterior proximity group
Table 2 Comparison of the sagittal balance parameters between the anterior and posterior proximity group

\begin{tabular}{lllll}
\hline & All $(n=47)$ & Anterior $(n=24)$ & Posterior $(n=23)$ & $p$ \\
\hline Male & 11 & $6(25 \%)$ & $5(22 \%)$ & \\
Female & 36 & $18(75 \%)$ & $18(78 \%)$ & \\
Age & $44.7 \pm 8.3$ & $45.7 \pm 8.6$ & $43.7 \pm 8.4$ & 0.419 \\
Height $(\mathrm{cm})$ & $161 \pm 7.5$ & $161.2 \pm 8.2$ & $160.6 \pm 7.0$ & 0.766 \\
Body weight $(\mathrm{kg})$ & $55.3 \pm 9.6$ & $56 \pm 9.2$ & $54.5 \pm 10.4$ & 0.601 \\
BMI & $21.3 \pm 3.1$ & $21.5 \pm 3$ & $21.1 \pm 3.4$ & 0.657 \\
\hline
\end{tabular}

BMI, body mass index

\begin{tabular}{lcccc}
\hline & All $(n=47)$ & Anterior $(n=24)$ & Posterior $(n=23)$ & $p$ \\
\hline SVA $(\mathrm{mm})$ & $17.7 \pm 17.2$ & $23.5 \pm 18.9$ & $11.6 \pm 13.5$ & $0.016^{*}$ \\
T1-Spi $\left(^{\circ}\right)$ & $-3.2 \pm 1.9$ & $-2.6 \pm 1.7$ & $-3.9 \pm 1.8$ & $0.015^{*}$ \\
TPA $\left(^{\circ}\right)$ & $12.1 \pm 5.4$ & $12.1 \pm 5.9$ & $12.0 \pm 5.1$ & 0.974 \\
PT $\left(^{\circ}\right)$ & $15.3 \pm 5.8$ & $14.7 \pm 6.1$ & $15.9 \pm 5.7$ & 0.469 \\
SS $\left(^{\circ}\right)$ & $33.8 \pm 6.4$ & $33.4 \pm 4.8$ & $34.2 \pm 7.9$ & 0.694 \\
PI $\left(^{\circ}\right)$ & $49.1 \pm 8.6$ & $48.1 \pm 7.2$ & $50.1 \pm 10.0$ & 0.433 \\
LL $\left(^{\circ}\right)$ & $43.3 \pm 8.9$ & $41.3 \pm 7.9$ & $45.3 \pm 9.8$ & 0.132 \\
TK $\left(^{\circ}\right)$ & $31.5 \pm 7.4$ & $30.4 \pm 8.2$ & $32.7 \pm 6.6$ & 0.287 \\
CAM-C7-offset $(\mathrm{mm})$ & $-5.8 \pm 8.2$ & $-7.3 \pm 7.6$ & $-4.3 \pm 8.8$ & 0.226 \\
CAM-HA-offset $(\mathrm{mm})$ & $15.1 \pm 18.7$ & $5.8 \pm 16.8$ & $24.7 \pm 15.6$ & $<0.000^{*}$ \\
\hline
\end{tabular}

$S V A$ sagittal vertical axis, $T 1-S P i \mathrm{~T} 1$ spinopelvic inclination, $T P A$ T1-pelvic angle, $P T$ pelvic tilt, $S S$ sacral slope, $P I$ pelvic incidence, $L L$ lumbar lordosis, $T K$ thoracic kyphosis, $C A M$ centre of the acoustic meati, CAM-C7-offset offset distance between the CAM and C7 plumb line, CAM-HA-offset offset distance between the CAM and hip axis 
Variations in standing sagittal alignment among healthy subjects have been documented [25]. Differences in the sagittal parameters were found between male and female volunteers with respect to their SVA, PT, angle pelvi-femoral, and knee flexion angle. In addition, an increase in cervical lordosis and TK along with a decrease in LL was significantly associated with ageing. The change in the spinal alignment by ageing (known as 'trunk stooping') is accompanied with an increase in PT, hip flexion, knee flexion, and ankle dorsiflexion, which are considered as compensation strategies by the lower extremity for trunk stooping. The postural change in the spine and lower extremity caused by ageing would be a crucial factor to change the loading of the hip joint. In particular, trunk stooping and an increase in hip flexion are likely to increase the anterior loading of the hip and may increase the risk of hip OA. We included healthy subjects aged $45 \pm 8$ years, and no difference was found between the anterior and posterior proximity groups in terms of age. Further analysis with a more aged population will explain the relation between postural change due to ageing and onset of hip OA.

The definition of 'natural' or 'comfortable' standing posture remains controversial. Recently, several alignment studies used the EOS system with the controlled standing position $[6,19]$. The standing position was standardised to either the 'hands on the cheeks' or 'hands on the clavicles' position. This is because both arms overlap on the spine in the lateral radiography, which possibly reduces the accuracy of the radiographic measurements. Zheng et al. evaluated the repeatability of the $\mathrm{C} 7$ plumb line and gravity line under three different arm positions (hands down, shoulder and elbow flexed at $45^{\circ}$ while holding a pole, and hands on the clavicles) while standing [27]. They found that the repeatability of the gravity line was better than that of the $\mathrm{C} 7$ plumb line, and there was an approximately $2-\mathrm{cm}$ posterior shift of the $\mathrm{C} 7$ plumb line in the clavicle position compared with the neutral (hands-down) position. The results of Zheng et al.'s study suggest that the arm position in standing can affect the sagittal spinal balance, and this limits the comparison of sagittal balance in our results with the resulted reported in other studies using EOS. In this study, the sagittal balance parameters and distance of hip joint space were analysed in the hands-down position as it is considered a physiological standing posture. The difference in the sagittal parameters due to the arm position while standing will be evaluated in a future study.

This study has several limitations to be addressed. First, the proximity area of the hip was defined as within $1 \mathrm{~mm}$ of the Hausdorff distance, whereas the 1-mm distance between the femoral head and acetabulum may not reflect the actual contact of the bones. Moreover, there was no previous definition of the hip joint contact area by distance, and the difference in cartilage thickness around the hip joint has been reported [28]. The choice of the threshold was experimental and lacks validation. As the in-plane resolution of the CT used in this study was $0.5 \mathrm{~mm} \times 0.5 \mathrm{~mm}$, the threshold discussion of every $0.5 \mathrm{~mm}$ was considered sufficient. This threshold was used to only identify trends in the proximity area, so it did not affect the final results to be evaluated in the closest area. Furthermore, an analysis was added to assess the subtle changes (e.g. $0.9 \mathrm{~mm}, 1.0 \mathrm{~mm}$, and $1.1 \mathrm{~mm}$ ). The subtle changes did not change the trend in the proximal area, and the results are shown in the supplementary figure (Fig. $\mathrm{S} 1)$. Further studies to obtain consensus from the other researchers and validate the relation between bone-to-bone distance and joint contact of the hip are warranted. Second, we excluded volunteers who had contact on the pole to avoid non-physiological standing position. This study criterion limits the number of volunteers, as we needed to reduce motion artefact and secure safety during 20-30 s of scanning time in the CT gantry. We used population values and the sigma of the previous study and calculated the sample size with a probability of 0.05 , effect size of 0.5 , and power of 0.8 . The analysis revealed that a minimum sample size of 44 was required for the study, so a total sample size of 47 cases was considered reasonable [10]. Third, this study did not consider lower extremity parameters and the morphology of the femur. This is because the movement of the CT gantry is limited to $40-175 \mathrm{~cm}$ from the floor, so CT images of the distal femur were not included in all the volunteers. Femoral anteversion is defined as the angle between an imaginary transverse line that runs medially to laterally through the knee joint and an imaginary transverse line passing through the centre of the femoral head and neck. Because of this limitation, femoral anteversion could not be measured. Fourth, this study did not clarify whether a change in the proximity area led to a change in the parameters or whether a change in the parameters led to a change in the proximity area. In order to show the causal relationship, it is necessary to reach a consensus on the measurement method and longitudinal study. If varying the standing position (e.g. bending forward and backward) in the same subject causes the changes in the proximity area, this may support the possibility that the parameters are the cause of these changes. However, considering the exposure to radiation, multiple scanning of healthy volunteers in a short period involves ethical issues.

This study was a preliminarily study using upright CT. This device was innovative, and the first goal was to identify trends in healthy volunteers. In the future, we plan to collect data on disease groups (e.g. limited OA and labrum tear, which are supposed to have instability) and compare the changes in data. 


\section{Conclusion}

Analysis of the sagittal balance and the distance of hip joint space revealed that the anterior proximity group had a positive anterior spinal balance and the posterior proximity group had a posterior gravity line compared with the hip joint centre. The results of this study suggest that the anterior spinal balance contributes to the anterior loading of the hip joint, which is known to be related to the initiation and onset of hip OA.

Funding This study was supported by Japan Society for the Promotion of Science (JSPS) KAKENHI (grant number 17H04266 and 20K08056), Uehara Memorial Foundation, and Canon Medical Systems (Otawara, Japan).

\section{Compliance with ethical standards}

Conflict of interest Masahiro Jinzaki has received a grant from Canon Medical Systems. However, Canon Medical Systems was not involved in the design and conduct of the study; in the collection, analysis, and interpretation of the data; or in the preparation, review, and approval of the manuscript. The remaining authors have no conflicts of interest to declare.

Open Access This article is licensed under a Creative Commons Attribution 4.0 International License, which permits use, sharing, adaptation, distribution and reproduction in any medium or format, as long as you give appropriate credit to the original author(s) and the source, provide a link to the Creative Commons licence, and indicate if changes were made. The images or other third party material in this article are included in the article's Creative Commons licence, unless indicated otherwise in a credit line to the material. If material is not included in the article's Creative Commons licence and your intended use is not permitted by statutory regulation or exceeds the permitted use, you will need to obtain permission directly from the copyright holder. To view a copy of this licence, visit http://creativecommons.org/licenses/by/4.0/.

\section{References}

1. Glassman SD, Bridwell K, Dimar JR, Horton W, Berven S, Schwab F (2005) The impact of positive sagittal balance in adult spinal deformity. Spine (Phila Pa 1976) 30:2024-2029. https:// doi.org/10.1097/01.brs.0000179086.30449.96

2. Lafage V, Schwab F, Skalli W, Hawkinson N, Gagey P, Ondra S, Farcy JP (2008) Standing balance and sagittal plane spinal deformity: analysis of spinopelvic and gravity line parameters. Spine (Phila Pa 1976) 33:1572-1578. https://doi.org/10.1097/ BRS.0b013e31817886a2

3. Devin CJ, McCullough KA, Morris BJ, Yates AJ, Kang JD (2012) Hip-spine syndrome. J Am Acad Orthop Surg 20:434-442. https ://doi.org/10.5435/JAAOS-20-07-434

4. Offierski CM, MacNab I (1983) Hip-spine syndrome. Spine (Phila Pa 1976) 8:316-321. https://doi.org/10.1097/00007632-19830 4000-00014

5. Buckland AJ, Vigdorchik J, Schwab FJ, Errico TJ, Lafage R, Ames C, Bess S, Smith J, Mundis GM, Lafage V (2015) Acetabular anteversion changes due to spinal deformity correction: bridging the gap between hip and spine surgeons. J Bone Joint Surg Am 97:1913-1920. https://doi.org/10.2106/JBJS.O.00276

6. Lazennec JY, Brusson A, Rousseau MA (2011) Hip-spine relations and sagittal balance clinical consequences. Eur Spine J 20(Suppl 5):686-698. https://doi.org/10.1007/s00586-011-1937-9

7. Okuda T, Fujita T, Kaneuji A, Miaki K, Yasuda Y, Matsumoto T (2007) Stage-specific sagittal spinopelvic alignment changes in osteoarthritis of the hip secondary to developmental hip dysplasia. Spine (Phila Pa 1976) 32:e816-e819. https://doi. org/10.1097/BRS.0b013e31815ce695

8. Weng WJ, Wang WJ, Wu MD, Xu ZH, Xu LL, Qiu Y (2015) Characteristics of sagittal spine-pelvis-leg alignment in patients with severe hip osteoarthritis. Eur Spine J 24:1228-1236. https ://doi.org/10.1007/s00586-014-3700-5

9. Yoshimoto H, Sato S, Masuda T, Kanno T, Shundo M, Hyakumachi T, Yanagibashi Y (2005) Spinopelvic alignment in patients with osteoarthrosis of the hip: a radiographic comparison to patients with low back pain. Spine (Phila Pa 1976) 30:1650-1657. https://doi.org/10.1097/01.brs.0000169446 .69758.fa

10. Day LM, DelSole EM, Beaubrun BM, Zhou PL, Moon JY, Tishelman JC, Vigdorchik JM, Schwarzkopf R, Lafage R, Lafage V, Protopsaltis T, Buckland AJ (2018) Radiological severity of hip osteoarthritis in patients with adult spinal deformity: the effect on spinopelvic and lower extremity compensatory mechanisms. Eur Spine J 27:2294-2302. https://doi.org/10.1007/s0058 6-018-5509-0

11. Ochi H, Homma Y, Baba T, Nojiri H, Matsumoto M, Kaneko K (2017) Sagittal spinopelvic alignment predicts hip function after total hip arthroplasty. Gait Posture 52:293-300. https://doi. org/10.1016/j.gaitpost.2016.12.010

12. Fukushima K, Miyagi M, Inoue G, Shirasawa E, Uchiyama K, Takahira N, Takaso M (2018) Relationship between spinal sagittal alignment and acetabular coverage: a patient-matched control study. Arch Orthop Trauma Surg 138:1495-1499. https://doi. org/10.1007/s00402-018-2992-z

13. Beck M, Kalhor M, Leunig M, Ganz R (2005) Hip morphology influences the pattern of damage to the acetabular cartilage: femoroacetabular impingement as a cause of early osteoarthritis of the hip. J Bone Joint Surg Br 87:1012-1018. https://doi. org/10.1302/0301-620X.87B7.15203

14. Leunig M, Beaule PE, Ganz R (2009) The concept of femoroacetabular impingement: current status and future perspectives. Clin Orthop Relat Res 467:616-622. https://doi.org/10.1007/s1199 9-008-0646-0

15. Eijer H, Hogervorst T (2017) Femoroacetabular impingement causes osteoarthritis of the hip by migration and micro-instability of the femoral head. Med Hypotheses 104:93-96. https://doi. org/10.1016/j.mehy.2017.05.035

16. Ganz R, Leunig M, Leunig-Ganz K, Harris WH (2008) The etiology of osteoarthritis of the hip: an integrated mechanical concept. Clin Orthop Relat Res 466:264-272. https://doi.org/10.1007/ s11999-007-0060-z

17. Hellman MD, Haughom BD, Brown NM, Fillingham YA, Philippon MJ, Nho SJ (2017) Femoroacetabular impingement and pelvic incidence: radiographic comparison to an asymptomatic control. Arthroscopy 33:545-550. https://doi.org/10.1016/j.arthr o.2016.08.033

18. Grammatopoulos G, Speirs AD, Ng KCG, Riviere C, Rakhra KS, Lamontagne M, Beaule PE (2018) Acetabular and spino-pelvic morphologies are different in subjects with symptomatic cam femoro-acetabular impingement. J Orthop Res 36:1840-1848. https://doi.org/10.1002/jor.23856

19. Bendaya S, Lazennec JY, Anglin C, Allena R, Sellam N, Thoumie P, Skalli W (2015) Healthy vs. osteoarthritic hips: a comparison of hip, pelvis and femoral parameters and relationships using the 
EOS® system. Clin Biomech (Bristol, Avon) 30:195-204. https ://doi.org/10.1016/j.clinbiomech.2014.11.010

20. Dubousset J, Charpak G, Dorion I, Skalli W, Lavaste F, Deguise J, Kalifa G, Ferey S (2005) A new 2D and 3D imaging approach to musculoskeletal physiology and pathology with low-dose radiation and the standing position: the EOS system. Bull Acad Natl Med 189:287-297 (discussion 297-300)

21. Jinzaki M, Yamada Y, Nagura T, Nakahara T, Yokoyama Y, Narita K, Ogihara N, Yamada M (2020) Development of upright computed tomography with area detector for whole-body scans: phantom study, efficacy on workflow, effect of gravity on human body, and potential clinical impact. Invest Radiol 55:73-83. https ://doi.org/10.1097/RLI.0000000000000603

22. Yamada Y, Yamada M, Yokoyama Y, Tanabe A, Matusoka S, Niijima Y, Narita K, Nakahara T, Murata M, Fukunaga K, Chubachi S, Jinzaki M (2020) Differences in lung and lobe volumes between supine and standing positions scanned with conventional and newly developed 320-detector-row upright CT: Intraindividual comparison. Respiration 99:598-605. https://doi. org/10.1159/000507265

23. Yamada Y, Jinzaki M, Hosokawa T, Tanami Y, Sugiura H, Abe T, Kuribayashi S (2012) Dose reduction in chest CT: comparison of the adaptive iterative dose reduction $3 \mathrm{D}$, adaptive iterative dose reduction, and filtered back projection reconstruction techniques. Eur J Radiol 81:4185-4195. https://doi.org/10.1016/j.ejrad .2012 .07 .013
24. Kurrat H, Oberländer W (1978) The thickness of the cartilage in the hip joint. J Anat 126:145-155

25. Hasegawa K, Okamoto M, Hatsushikano S, Shimoda H, Ono M, Homma T, Watanabe K (2017) Standing sagittal alignment of the whole axial skeleton with reference to the gravity line in humans. J Anat 230:619-630. https://doi.org/10.1111/joa.12586

26. Lewis CL, Sahrmann SA, Moran DW (2007) Anterior hip joint force increases with hip extension, decreased gluteal force, or decreased iliopsoas force. J Biomech 40:3725-3731. https://doi. org/10.1016/j.jbiomech.2007.06.024

27. Zheng X, Chaudhari R, Wu C, Mehbod AA, Transfeldt EE, Winter RB (2010) Repeatability test of C7 plumb line and gravity line on asymptomatic volunteers using an optical measurement technique. Spine (Phila Pa 1976) 35:E889-E894. https://doi.org/10.1097/ brs.0b013e3181db7432

28. Mechlenburg I, Nyengaard JR, Gelineck J, Soballe K, Troelsen A (2010) Cartilage thickness in the hip measured by MRI and stereology before and after periacetabular osteotomy. Clin Orthop Relat Res 468:1884-1890. https://doi.org/10.1007/s1199 9-010-1310-z

Publisher's Note Springer Nature remains neutral with regard to jurisdictional claims in published maps and institutional affiliations. 